\title{
Do patients with obstructive sleep apnoea deserve new dedicated antihypertensive strategies?
}

\author{
Renaud Tamisier, ${ }^{1,2}$ Patrick Lévy, ${ }^{1,2}$ Jean-Louis Pépin ${ }^{1,2}$
}

Hypertension affects 25\% of the adult population and remains a leading cause of cardiovascular mortality, accounting for $13.5 \%$ of all deaths. Half of all strokes and ischaemic heart disease events are attributed to hypertension. ${ }^{1}{ }^{2}$ Most patients exhibit grade I hypertension (systolic blood pressure of $140-159 \mathrm{~mm} \mathrm{Hg}$ and/or diastolic blood pressure of 90$99 \mathrm{~mm} \mathrm{Hg}$ ) without coexisting cardiovascular disease. Effective reduction of blood pressure in this population significantly reduces stroke and death. ${ }^{3}$

Obstructive sleep apnoea (OSA) is now recognised as a risk factor for the development of hypertension in European and the US International Guidelines. In OSA, elevation of blood pressure is in part due to intermittent hypoxaemia leading to increased sympathetic tone and impaired baroreflex gain. ${ }^{4}$ Altered arterial vasoconstriction and vasodilatation $^{5}$ owing to stimulation of the renin-angiotensin-aldosterone system (RAAS) $^{6}$ are also significant contributors. Although OSA and hypertension are tightly linked in a dose-response manner, the impact of short-term OSA treatment on blood pressure reduction in unselected patient populations with OSA is rather mild, about $2 \mathrm{~mm} \mathrm{Hg}$ reduction in 24-hour mean blood pressure. This effect is slightly greater in patients who are compliant with CPAP or mandibular advancement devices, presumably by allowing rapid eye movement (REM) sleep at the end of the night to be free of obstructive respiratory events. ${ }^{7-9}$

It stands to reason that treatment strategies combining OSA treatment and pharmacological antihypertensives would be synergistic. ${ }^{10} 11$ Previous studies ${ }^{10} 11$ have demonstrated that in hypertensive patients with OSA, losartan or valsartan are by far more effective than CPAP in reducing blood pressure, even if nocturnal blood pressure is better controlled when CPAP is used concurrently with these medications. This underlines the need for specific

${ }^{1}$ HP2 Laboratory, Inserm, Univ. Grenoble Alpes, Grenoble, France; ${ }^{2}$ Pôle Thorax et Vaisseaux, Grenoble Alpes University Hospital, Grenoble, France

Correspondence to Dr Renaud Tamisier, Laboratoire EFCR, CHU de Grenoble, CS 10217, 38043 Grenoble cedex 09, France; rtamisier@chu-grenoble.fr strategies to be further studied in hypertensive patients with OSA, as a distinct response is expected compared with the general non-OSA hypertensive population.

The study presented in this issue of Thorax by Serinel et al ${ }^{12}$ attempted to address one piece of the puzzle regarding a better strategy for managing OSA-related hypertension. Using a wellestablished cross-over randomised controlled trial design, the authors explored whether chronotherapy would be of particular benefit in hypertensive patients with OSA. As hypertension in OSA is classically nocturnal and aggravates during REM sleep, the authors hypothesised that the decrease in nocturnal blood pressure would be superior by administrating perindopril in the evening compared with in the morning. Chronotherapy in essential hypertension $^{13}$ or in hypertensive diabetics $^{14}$ improves the blood pressurelowering effect of pharmacotherapy during the night without weakening its daytime effect. The goal of administering antihypertensives in the evening is to restore the normal circadian dipping profile of blood pressure, which is the decrease of $>10 \%$ in sleep-time compared with daytime blood pressure during wakefulness. In the present study, however, an evening administration of perindopril did not lead to a superior effect on blood pressure control compared with morning administration in hypertensive patients with untreated moderate-to-severe OSA. In fact, daytime systolic blood pressure was more effectively reduced with morning than with evening administration of perindopril. These results highlight the complexities and differences when treating hypertension in patients with OSA. Chronotherapy alone is not adequate to improve nocturnal hypertension and it actually leads to poorer blood pressure control during daytime in this patient population. In the last part of the study, both groups were using CPAP for 8 weeks in an open-label design. This combined treatment (ie, ACE inhibitors and CPAP) further reduced the systolic blood pressure by an additional $3 \mathrm{~mm} \mathrm{Hg}$ during sleep, which confirms the findings of previous reports. ${ }^{10} 11$
The study by Serinel $e t a l^{12}$ is a wellconducted and analysed RCT. Some limitations may be the absence of a non-OSA hypertensive group and not using sham-CPAP, although these might have had only a marginal impact on the reported results. Moreover, it remains unclear if the negative results of chronotherapy with ACE inhibitors is a class-effect or applicable to all antihypertensives. Ultimately, a major limitation in the field of sleep apnoea and hypertension, which is not unique to the study by Serinel et al, is the relatively small sample sizes and the short-duration of follow-up $(n=79$ and follow-up of 6 weeks in the study by Serinel et al). This is far less than the 3013 patients included in the MAPEC study who were followed for a median of 5.6 years. The MAPEC investigators found a significant reduction in cardiovascular risk using evening administration of antihypertensives. ${ }^{15}$ The OSA community must convince academic and industrial funders of the rationale for defining specific indications for medications in OSA-related hypertension.

A major concept arising from the study by Serinel et al, and previous ones in this field is that OSA-related hypertension is a true 'resistant hypertension' that requires dedicated studies and specific guidelines. For example, a non-dipping profile is typical in these patients and such a profile, even in isolation, is related to a higher risk of cardiovascular disease and death. ${ }^{16}$ In the MAPEC study, chronotherapy improved nocturnal blood pressure and led to a $17 \%$ reduction in cardiovascular risk for each $5 \mathrm{~mm} \mathrm{Hg}$ reduction in systolic blood pressure during sleep $(p<0.001)$ in patients with essential hypertension. ${ }^{15}$ The negative findings by Serinel et $a l^{12}$ demonstrate that such results cannot be extrapolated to the OSA population. While CPAP is the gold standard treatment for OSA, it is not necessarily effective in reducing blood pressure, particularly if adherence to treatment is low. In the Sleep Apnea CardioVascular Endpoints (SAVE) study, there was no difference in cardiovascular outcomes in patients with OSA and established coronary or cerebrovascular disease with a mean duration of CPAP treatment of 3.7 years, compared with usual care. Moreover, mean CPAP adherence of 3.3 hours per night did not lead to any improvement in office arterial blood pressure. ${ }^{17}$ Taken together, these results emphasise the need to rethink therapeutic approaches in order to effectively reduce the blood pressure in different subpopulations of patients with OSA. Intrinsic and extrinsic factors that 


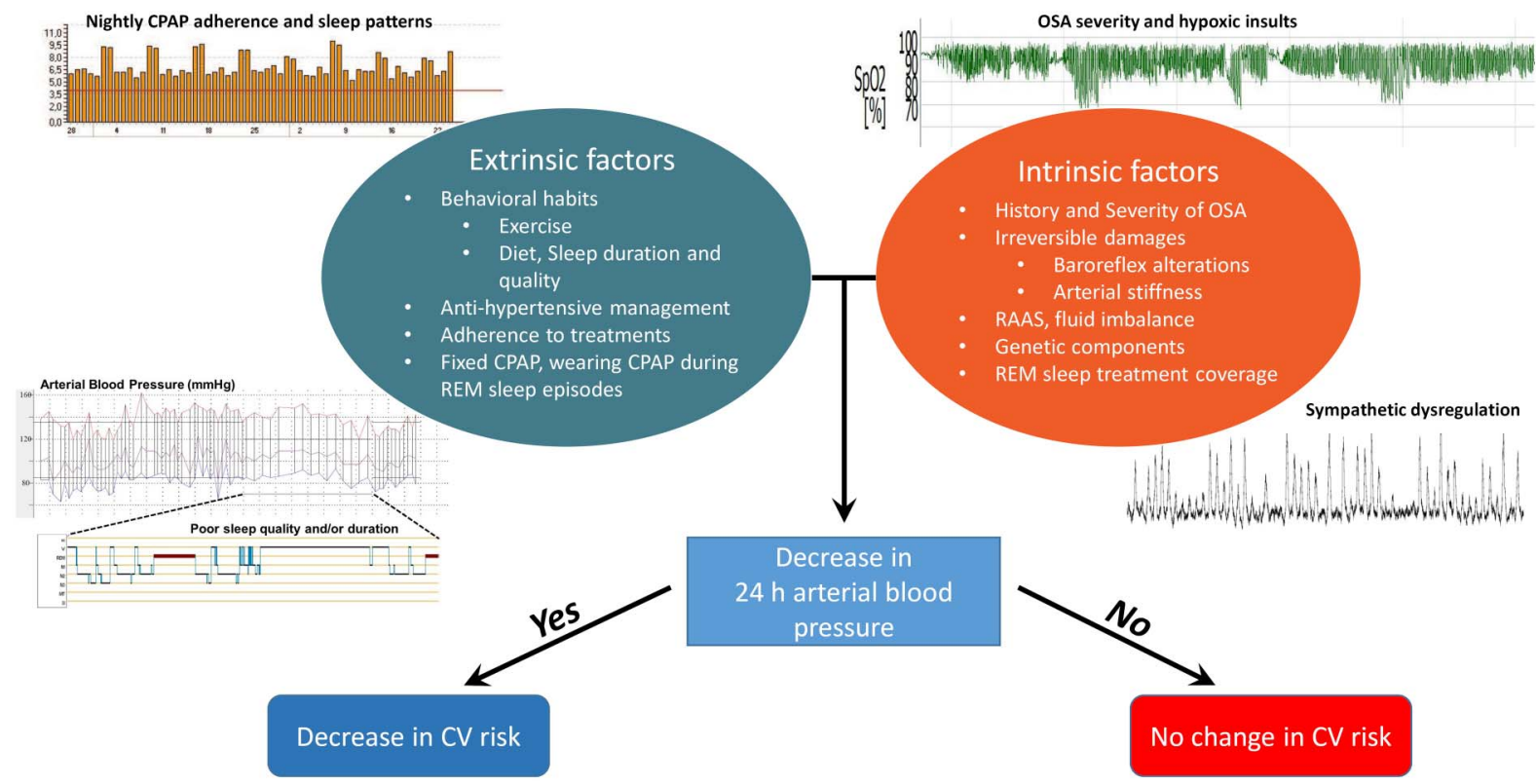

Figure 1 The pathophysiology of OSA-related hypertension. These intrinsic and extrinsic factors may influence the management of hypertension in patients with OSA. OSA severity and duration before diagnosis may have induced irreversible damage to the regulation of blood pressure and the vascular system. RAAS and sympathetic regulation of blood pressure are altered in hypertensive patients with OSA. These pathways could be targeted using antihypertensive drugs as is recommended for diabetics and patients with chronic kidney failure. We need to better identify blood pressure phenotype clusters in order to personalise therapy and ideally to predict response to different treatment strategies. ${ }^{23} \mathrm{CV}$, cardiovascular; OSA, obstructive sleep apnoea; RAAS, renin-angiotensin-aldosterone system; REM, rapid eye movement.

influence daytime and night-time blood pressure need to be taken into account in order to design effective strategies for lowering blood pressure in hypertensive patients with OSA (see figure 1). We recently reported that the level of physical activity, as measured by steps per day, was a significant determinant of daytime blood pressure, while the severity of oxygen desaturation during sleep was correlated with morning blood pressure. ${ }^{18}$ Accordingly, there is a need for clinical research studies that combine and compare various approaches such as CPAP, medications and physical activity (eg, the RAP clinical trial NCT02057783). Furthermore, interventions that can effectively improve CPAP adherence are essential. A recent report in Thorax showed that a better control of 24-hour blood pressure might be obtained by using fixed-pressure CPAP instead of auto-adjusting $\mathrm{CPAP},{ }^{19}$ and that wearing CPAP during the majority of REM sleep episodes at the end of the night is central for long-term control of blood pressure. ${ }^{8}$ Improving CPAP adherence remains a major goal, as studied in an ongoing trial of auto-adjusting CPAP with a pressure relief technology during periods of nighttime wakefulness (NCT02721329).

Surprisingly, although OSA is increasingly recognised as a major chronic disease, ${ }^{20}$ with hypertension as a comorbidity in half of patients with OSA, no specific recommendations are available in the international hypertension guidelines except for CPAP therapy. Even in well-defined populations like those with resistant hypertension and OSA, the blood pressure response to CPAP therapy is highly variable and unpredictable. As such, a combination of predictive biomarkers would be particularly helpful to guide clinicians in the management of asymptomatic or minimally symptomatic patients with OSA who are reluctant to accept long-term CPAP therapy. ${ }^{21} 22$

Acknowledgements We thank Alison Foote PhD (Grenoble Alps University Hospital) for critically editing the manuscript.

Funding This work is partly supported by the French National Research Agency in the framework of the "Investissements d'avenir" program (ANR-15-IDEX-02) and by Fond de dotation Agir pour les maladies chroniques.

Competing interests None declared.

Provenance and peer review Commissioned; internally peer reviewed.

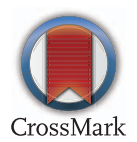

To cite Tamisier R, Lévy P, Pépin J-L. Thorax 2017;72:495-497.

Published Online First 6 March 2017

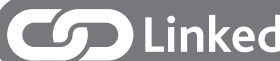

http://dx.doi.org/10.1136/thoraxjnl-2016-209504

Thorax 2017:72:495-497.

doi:10.1136/thoraxjnl-2016-209772

\section{REFERENCES}

1 Kearney PM, Whelton M, Reynolds K, et al. Global burden of hypertension: analysis of worldwide data. Lancet 2005;365:217-23.

2 Lawes CM, Vander Hoorn S, Rodgers A. Global burden of blood-pressure-related disease, 2001. Lancet 2008:371:1513-18.

3 Sundstrom J, Arima $H$, Jackson $R$, et al. Effects of blood pressure reduction in mild hypertension: a systematic review and meta-analysis. Ann Intern Med 2015;162:184-91.

4 Tamisier R, Pepin JL, Remy J, et al. 14 nights of intermittent hypoxia elevate daytime blood pressure and sympathetic activity in healthy humans. Eur Respir I 2011:37:119-28.

5 Grote L, Kraiczi H, Hedner J. Reduced alpha- and beta(2)-adrenergic vascular response in patients with obstructive sleep apnea. Am J Respir Crit Care Med 2000;162:1480-7.

6 Lacedonia D, Tamisier R, Roche F, et al. Respective effects of OSA treatment and angiotensin receptor blocker on aldosterone in hypertensive OSA patients: a randomized cross-over controlled trial. Int I Cardiol 2014;177:629-31.

7 Bratton DJ, Stradling JR, Barbe F, et al. Effect of CPAP on blood pressure in patients with minimally symptomatic obstructive sleep apnoea: a meta-analysis using individual patient data from four randomised controlled trials. Thorax 2014;69:1128-35.

8 Mokhlesi B, Hagen EW, Finn LA, et al. Obstructive sleep apnoea during REM sleep and incident non-dipping of nocturnal blood pressure: a longitudinal analysis of the Wisconsin Sleep Cohort. Thorax 2015;70:1062-9.

9 Bratton DJ, Gaisl T, Wons AM, et al. CPAP vs mandibular advancement devices and blood pressure 
in patients with obstructive sleep apnea: a systematic review and meta-analysis. JAMA 2015;314:2280-93.

10 Pepin JL, Tamisier R, Barone-Rochette G, et al. Comparison of continuous positive airway pressure and valsartan in hypertensive patients with sleep apnea. Am J Respir Crit Care Med 2010;182:954-60.

11 Thunstrom E, Manhem K, Rosengren A, et al. Blood pressure response to losartan and continuous positive airway pressure in hypertension and obstructive sleep apnea. Am J Respir Crit Care Med 2016;193:310-20.

12 Serinel Y, Yee BI, Grunstein RR, et al. Chronotherapy for hypertension in obstructive sleep apnoea (CHOSA): a randomized, double-blind, placebo controlled crossover trial. Thorax Published Online First: 14 Dec 2016. doi:10.1136/thoraxjnl-2016209504

13 Hermida RC, Ayala DE, Mojon A, et al. Role of time-of-day of hypertension treatment on the
J-shaped relationship between blood pressure and cardiovascular risk. Chronobiol Int 2013;30:328-39.

14 Hermida RC, Ayala DE, Mojon A, et al. Influence of time of day of blood pressure-lowering treatment on cardiovascular risk in hypertensive patients with type 2 diabetes. Diabetes Care 2011;34:1270-6.

15 Hermida RC, Ayala DE, Mojon A, et al. Decreasing sleep-time blood pressure determined by ambulatory monitoring reduces cardiovascular risk. J Am Coll Cardiol 2011:58:1165-73.

16 Boggia J, Li Y, Thijs L, et al. Prognostic accuracy of day versus night ambulatory blood pressure: a cohort study. Lancet 2007;370:1219-29.

17 McEvoy RD, Antic NA, Heeley E, et al. CPAP for prevention of cardiovascular events in obstructive sleep apnea. N Engl J Med 2016;375:919-31.

18 Mendelson M, Tamisier R, Laplaud D, et al. Low physical activity is a determinant for elevated blood pressure in high cardiovascular risk obstructive sleep apnea. Respir Care 2014;59:1218-27.
19 Pepin JL, Tamisier R, Baguet JP, et al. Fixed-pressure CPAP versus auto-adjusting CPAP: comparison of efficacy on blood pressure in obstructive sleep apnoea, a randomised clinical trial. Thorax 2016;71:726-33.

20 Levy P, Kohler M, McNicholas WT, et al. Obstructive sleep apnoea syndrome. Nat Rev Dis Primers 2015;1:15015.

21 Sanchez-de-la-Torre M, Khalyfa A, Sanchez-de-la-Torre A, et al. Precision medicine in patients with resistant hypertension and obstructive sleep apnea: blood pressure response to continuous positive airway pressure treatment. J Am Coll Cardiol 2015;66:1023-32.

22 Martinez-Garcia MA, Capote F, Campos-Rodriguez F, et al. Effect of CPAP on blood pressure in patients with obstructive sleep apnea and resistant hypertension: the HIPARCO randomized clinical trial. JAMA 2013;310:2407-15.

23 Bailly S, Destors M, Grillet $Y$, et al. Obstructive sleep apnea: a cluster analysis at time of diagnosis. PLOS ONE 2016:11:e0157318. 